\title{
Inseparability of science history and discovery
}

\author{
J. M. Herndon \\ Transdyne Corporation, 11044 Red Rock Drive, San Diego, CA 92131, USA
}

Received: 19 February 2010 - Accepted: 5 April 2010 - Published: 12 April 2010

\begin{abstract}
Science is very much a logical progression through time. Progressing along a logical path of discovery is rather like following a path through the wilderness. Occasionally the path splits, presenting a choice; the correct logical interpretation leads to further progress, the wrong choice leads to confusion. By considering deeply the relevant science history, one might begin to recognize past faltering in the logical progression of observations and ideas and, perhaps then, to discover new, more precise understanding. The following specific examples of science faltering are described from a historical perspective: (1) Composition of the Earth's inner core; (2) Giant planet internal energy production; (3) Physical impossibility of Earth-core convection and Earth-mantle convection, and; (4) Thermonuclear ignition of stars. For each example, a revised logical progression is described, leading, respectively, to: (1) Understanding the endo-Earth's composition; (2) The concept of nuclear georeactor origin of geo- and planetary magnetic fields; (3) The invalidation and replacement of plate tectonics; and, (4) Understanding the basis for the observed distribution of luminous stars in galaxies. These revised logical progressions clearly show the inseparability of science history and discovery. A different and more fundamental approach to making scientific discoveries than the frequently discussed variants of the scientific method is this: An individual ponders and through tedious efforts arranges seemingly unrelated observations into a logical sequence in the mind so that causal relationships become evident and new understanding emerges, showing the path for new observations, for new experiments, for new theoretical considerations, and for new discoveries. Science history is rich in "seemingly unrelated observations" just waiting to be logically and causally related to reveal new discoveries.
\end{abstract}

\section{Introduction}

Division and progressive subdivision with specialization comprise an integral process in nature and in human activity. Indeed, each of us began as a single cell which divided and progressively subdivided while achieving specialized functions. Ever-burgeoning observation, experimentation, derivation, calculation, and understanding, out of necessity, have led to division, progressive subdivision, and specialization of knowledge. By the 17th century, chemistry was developing its distinction as a clearly separate science from physics. Then, in the 20th century, as academicians expanded study of the Earth, those same divisions were carried forward as geochemistry and geophysics.

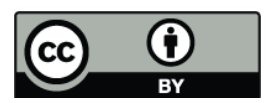

Correspondence to: J. M. Herndon (mherndon@san.rr.com)
But there is a problem: As geochemistry and geophysics are only partial descriptions of the Earth, their separation and specialization poses a serious impediment to understanding, and, consequently, to making important new discoveries, particularly in instances when geochemists have little training in physics and when geophysicists have little training in chemistry. Another, sometimes even more serious impediment to making important new discoveries, and one often least appreciated, arises as a consequence of excluding, from the realm of scientific investigation, understanding of relevant science history.

Science is very much a logical progression through time. Advances are frequently underpinned by ideas and understandings developed in the past, sometimes under circumstances which may no longer hold the same degree of validity. It is of great benefit for a scientist, working within a conceptual framework, to understand the historical basis of that framework, to understand how the present state of knowledge arose and under what circumstances. All too often, scientists, being distinctly human creatures of habit, plod optimistically along through time, eagerly looking toward the future, but 
rarely looking with question at circumstances from the past which have set them upon their present courses. Progressing along a logical path of discovery is rather like following a path through the wilderness. Occasionally, one comes to a juncture, the path splits, presenting a choice of scientific interpretations. Choose the correct logical interpretation and the way is clear for further progress; the wrong choice leads to confusion. That is often the way of science. To make matters even more complicated, the correct path is sometimes invisible, obscured because some requisite discovery has not yet been made. Moreover, the logical progression of scientific discovery is often opposed by the darker elements of human nature and institutional self-interest.

In 1623, Galileo Galilei (1564-1642), one of the greatest scientists of the millennium, precisely characterized human response to new ideas in a letter written to Don Virginio Cesarini, stating in part, "I have never understood, Your Excellency, why it is that every one of the studies I have published in order to please or to serve other people has aroused in some men a certain perverse urge to detract, steal, or deprecate that modicum of merit which I thought I had earned, if not for my work, at least for its intention" (Drake, 1956).

Much has been written about the Roman Catholic Church's opposition to the heliocentric hypothesis of Nicolaus Copernicus (1473-1543) and its consequences on individuals and on the progression of human knowledge; see, for example (Stimson, 1917). Less known, though, is that about 1800 years before Copernicus (1543), Aristarchus of Samos (310-230 BC) had arrived at the same idea. Although the original explanatory document is lost, clear reference is given to his ideas by Archimedes (287-213 BC) in his book "The Sand Reckoner" which states in part, "His hypotheses are that the fixed stars and the sun remain unmoved, that the earth revolves about the sun in the circumference of a circle, the sun lying in the middle of the orbit, and that the sphere of fixed stars, situated about the same center as the sun ..." (Heath, 1897).

What, one might logically ask, is the relevancy of the above historical references, especially now in the time of near-instantaneous global communications and Internet access? The relevancy relates to the persistence of human nature, which does not change on a time-scale of a few hundreds or even a few thousands of years, and which underlies impediments posed by institutional self-interest.

Science is like a long road paved with observations, ideas, and understandings. From a distance it might seem like a smooth strip of ribbon meandering through time. But up close, it can be seen as a rocky road indeed - a mix of insight and oversight, design and serendipity, precision and error, and implication and revision, all too often influenced by the vagaries of human behavior. By considering deeply the relevant science history, one might begin to recognize past faltering in the logical progression of observations and ideas and, perhaps then, to discover new, more precise understanding. While seeming abstract, these introductory consid- erations are concretely illustrated in the following examples from recent-history developments in geo- and space sciences, and clearly show the inseparability of science history and discovery.

\section{Logical juncture: composition of the Earth's inner core}

The Law of Universal Gravitation, formulated by Isaac Newton (1643-1727), provided a mathematical basis for determining the mass of the Earth, but it could not be applied until a calibration factor, the Universal Gravitational Constant, was experimentally determined by measuring the gravitational attraction between two known masses. Henry Cavendish (1731-1810), using a torsion balance invented and fabricated by John Mitchell (1724-1793), made the first determination of the mean density of the Earth (Cavendish, 1798). Interestingly, Cavendish did not set out explicitly to measure the Universal Gravitational Constant, which can be determined from his data, but rather to measure the density of the Earth, which he found to be $5.48 \mathrm{~g} / \mathrm{cm}^{3}$, a result quite close to the modern measured value of $5.53 \mathrm{~g} / \mathrm{cm}^{3}$. But, one hundred years would elapse before the significance of Cavendish's density measurement would be realized and would begin to indicate Earth's actual composition.

The German seismologist, Emil Wiechert (1861-1928), lived during a time of extensive industrial iron-making in Europe and was acquainted with the process of molten iron metal settling beneath slag. He had seen nickeliferous iron meteorites in museums, as well as stone and stony-iron meteorites. Realizing that the mean density of Earth, as measured by Cavendish, is too great for the Earth to consist entirely of rock, Wiechert suggested that the Earth has at its center a core of iron metal, like the metal of iron meteorites (Wiechert, 1897). Less than a decade would elapse before that idea would be validated with the discovery of the Earth's core.

The English seismologist, Richard D. Oldham (18581936), after serving as Director of the Geological Survey of India, was investigating the speed of earthquake waves as a function of their depth within the Earth. Oldham found that the speed of earthquake waves becomes faster with greater depth of penetration into the Earth, but only to a certain depth. Below that depth, earthquake waves suddenly travel much slower, indicating a profoundly different material. Oldham (1906) thus discovered that the Earth does in fact have a core, just as Wiechert (1897) had envisioned might be the case. Over the next two decades, the size of the Earth's core was determined with precision and its physical state was shown to be liquid, as transverse (shear) earthquake waves could not pass through it (Jeffreys, 1929). 


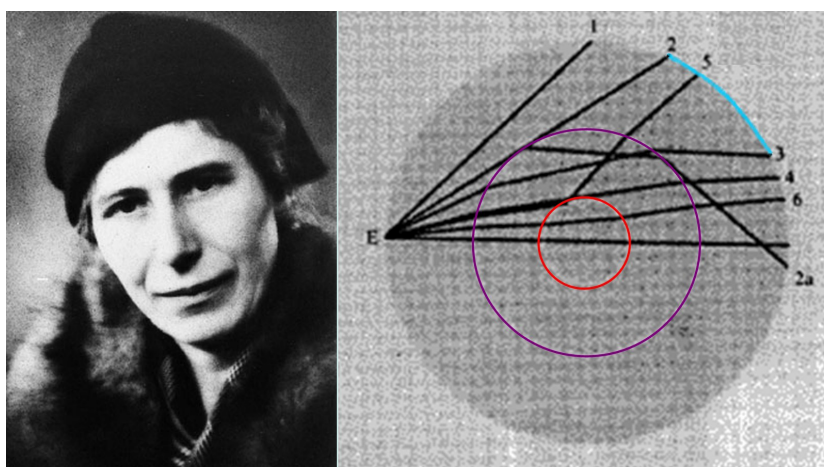

Figure 1. Photograph of Inge Lehmann beside a scan of her original diagram showing discovery of the Earth's inner core (Lehmann, 1936). For improved clarity, the circles representing the inner core and the fluid core have subsequently been traced over in red and purple, respectively. The shadow zone, not specifically marked on her original diagram, is indicated in blue. Note the reflection of ray \#5 into the shadow zone.

During the same period of time, numerous chemical analyses of meteorites and spectroscopic analyses of stars were being made and tabulated (Goldschmidt, 1937, 1938). The data obtained not only justified Wiechert's assertion of the Earth being similar to a meteorite (Wiechert, 1897), but would eventually become crucial for understanding the internal composition of the Earth.

By the early 1930s, the structure of the Earth was thought to be simple, consisting of just the fluid core, surrounded by a uniform shell of solid rock, called the mantle, and topped by a very thin crust, which had been discovered in 1909 by the Croatian seismologist, Andrija Mohorovičić (1857-1936). But then, seismologists, analyzing the seismograph records of a large earthquake that had occurred in 1929 near New Zealand, discovered a mystery: Earthquake waves change speed and direction as they enter a different material at an angle. As a consequence, there is a "shadow zone" where earthquake waves should not have been detected, but in this instance were indeed recorded. After much discussion and debate, in 1936 the Danish seismologist, Inge Lehmann (18881993), solved that mystery. She had the idea, as illustrated in Fig. 1, that within the fluid core there might be a small, solid inner core which would reflect earthquake waves into the shadow zone (Lehmann, 1936).

Solving the shadow zone mystery opened yet another: What then is the chemical composition of the inner core? Seismic data, coupled with moment of inertia considerations, can yield information on the existence of physical structures within the Earth and whether they are solid or liquid, but not their chemical compositions, which must come from implications derived from meteorites. The fundamental relationships connecting the isotope-compositions of the elements of Earth with those of the chondrite-meteorites, and connecting the abundances of the non-gaseous chemical elements of chondrite-meteorites with corresponding abundances of the elements in the outer portion of the Sun form the basis for knowledge of the composition of the Earth as a whole (Aller, 1961; Anders and Grevesse, 1989; Suess and Urey, 1956). But, there is a serious complication; not all chondritemeteorites are identical (Mason, 1962; Scott, 2007; StoryMaskelyne, 1870). And, although the differences are small, in certain respects they are profound (Herndon, 2008).

About $90 \%$ of the meteorites that are observed falling to Earth are called ordinary chondrites, because they are so common. These are composed of iron metal and silicaterock, along with some iron sulfide. Many scientists, including the renowned Harvard Professor, Francis Birch (19031992), assumed that the Earth as a whole is like an ordinary chondrite (Birch, 1964; Daly, 1943), which would seem to account for the Earth having an iron alloy core surrounded by a silicate mantle.

Only five chemical elements, iron, magnesium, silicon, oxygen, and sulfur, account for about $95 \%$ of the mass of a chondrite-meteorite; $98 \%$, if the four minor elements, nickel, calcium, aluminum, and sodium, are included (Herndon, 1998). Birch realized, as did some others, that iron and nickel occur alloyed together in ordinary chondrite metal and in other instances where meteoritic metal had been analyzed. Moreover, the sum of abundances of all the elements heavier than nickel is insufficiently great to account for mass of the inner core. To Birch the answer was clear. Earth's core must be iron in the process of freezing, the inner core being the frozen solid iron, while the surrounding fluid core is iron that is still liquid (Birch, 1940, 1952, 1972).

Birch was progressing along a logical path of discovery, proceeding beyond Lehmann (1936) and came to a juncture, a place where the path splits. At the time, though, both the juncture and the correct path were invisible. The observations required for understanding and to reveal the invisible path would come decades later, not from studying ordinary chondrites, but from investigating enstatite chondrites, the rare group of chondrite-meteorites that Birch had ignored. In the meantime, geoscientists proceeded forward on an illogical path which could not, and would not, lead to important new discoveries.

\section{Invisible path at logical juncture: the nickel silicide inner core of the Earth}

In the 1960s, two decades after Birch (1940) had pronounced the inner core to be partially crystallized iron metal, silicon was discovered in the metal of some enstatite chondrites (Ringwood, 1961). Also, in the 1960s, a new mineral was discovered in enstatite chondrites, which consists of the elements nickel and silicon, called nickel silicide or the mineral name, perryite. Meteoritic nickel silicide occurs both as lamellar exsolutions from silicon-bearing iron metal (Fredriksson and Henderson, 1965; Ramdohr, 1964; 
Ramdohr and Kullerud, 1962; Wai, 1970; Wasson and Wai, 1970) and as more massive forms intimately associated with metal and iron sulfide in certain enstatite chondrites (Ramdohr, 1973; Reed, 1968). Yet another decade would elapse before the significance of those observations would be appreciated by the American physicist/chemist, J. Marvin Herndon.

Birch had thought that nickel and iron were always alloyed and were essentially inseparable in meteoritic metal, but clearly that was not the case in the oxygen-poor enstatite chondrite matter. What Herndon (1979) realized and published in the "Proceedings of the Royal Society of London" was that, if silicon were present in the fluid core of the Earth, under appropriate conditions, the silicon could combine with nickel, forming a compound called nickel silicide, which could be solid and denser than the iron-sulfur alloy fluid core. The precipitated nickel silicide would then settle to the center by gravity and would comprise a mass virtually identical to the mass of the inner core. The existence of the nickel silicide inner core would mean that the interior of the Earth was formed from primordial matter which was sufficiently oxygen-poor as to have caused elemental silicon to be present in the iron liquid. In other words, the deep interior of Earth would have to be like an enstatite chondrite, not like an ordinary chondrite, as many before had thought.

The purpose of science is to understand the true nature of Earth and Universe. Thus, when a major contradiction to an important concept arises, there should be discussion and debate. Every effort should be made either to confirm or to refute the concept, by experiment or by calculation. If the new concept is found to be in error, it should be refuted, preferably in the journal of original publication; otherwise it should be acknowledged in the scientific literature. Herndon's nickel silicide inner core concept has never been refuted in the scientific literature and no attempt has been made to experimentally ascertain phase relations at inner-core pressures and temperatures (Herndon, 1998).

As a matter of historical interest, in a letter to Herndon, dated 17 August 1979 and shown in Fig. 2, Inge Lehmann stated in part, "I admire the precision of your reasoning based upon available information, and I congratulate you on the highly important result you have obtained."

\section{Beginning anew the logical progression of understanding and discovery: composition of the Earth's interior}

Believing that the inside of Earth is like an ordinary chondrite meteorite, as many did, leads to the conclusion that the silicate-rock mantle must be made of only one kind of rock below the crust and above the core. But, as early as the late 1930s, the New Zealand-born, Australian seismologist, Keith Edward Bullen (1906-1976), had discovered that the silicate-rock mantle is not featureless, as it first had seemed

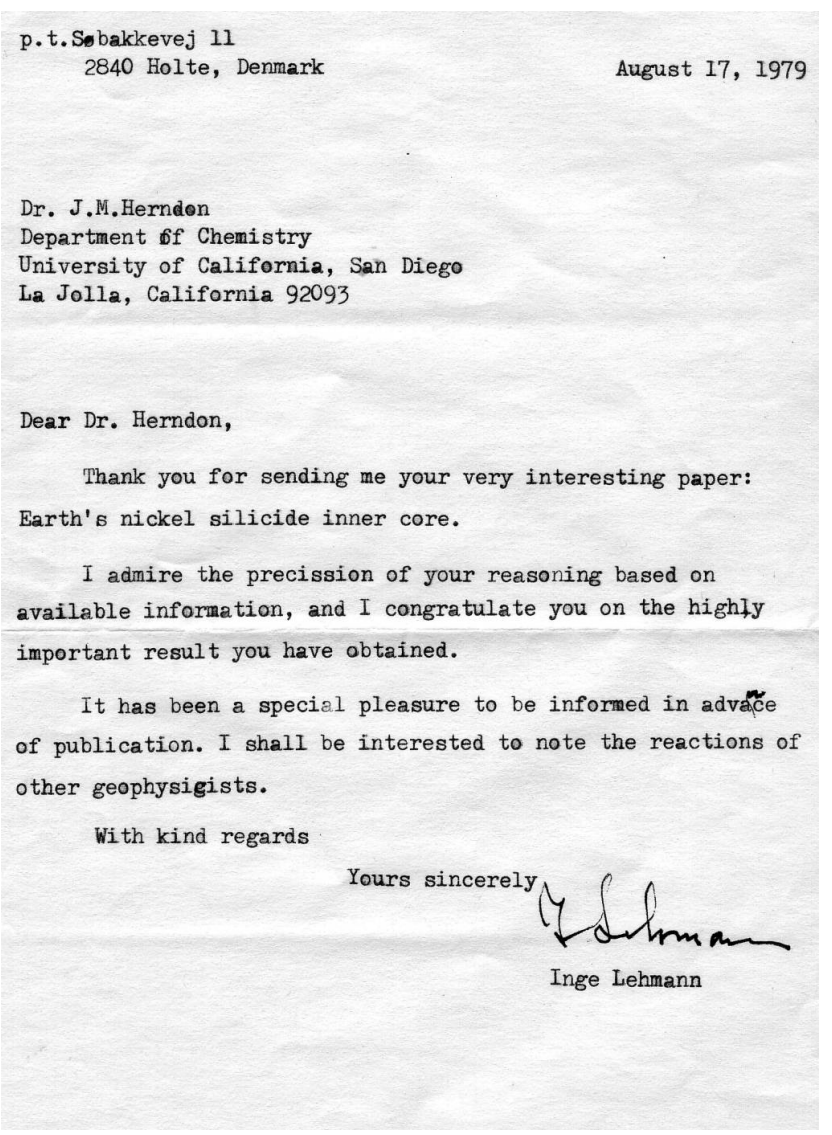

Figure 2. Scan of the congratulatory letter Inge Lehmann sent to J. Marvin Herndon.

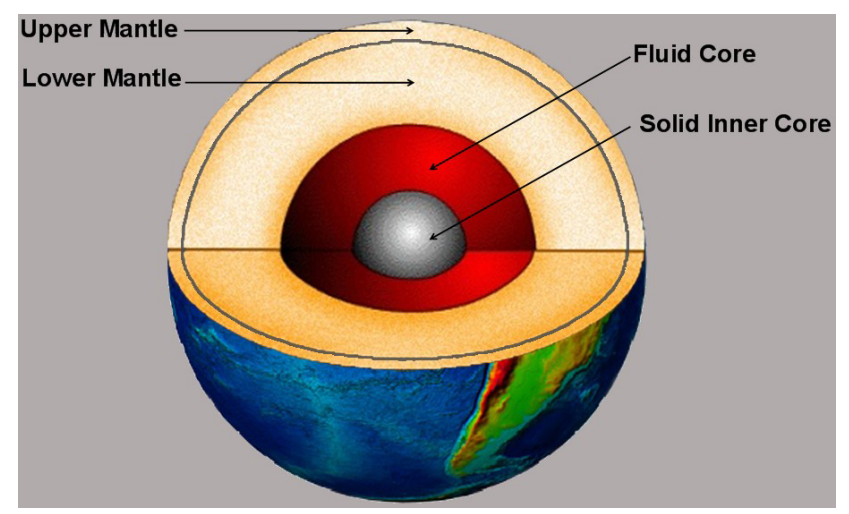

Figure 3. Schematic representation of the major parts of the Earth. From Herndon (2008).

(Bullen, 1938, 1946). Bullen discovered that there is a seismic boundary or "discontinuity" which separates the mantle into two major parts as illustrated in Fig. 3. Below that boundary, the lower mantle, as it now called, comprises about $49 \%$ of the mass of the Earth and is without seismic irregularities down to the boundary of the core. The upper mantle, lying above that boundary and comprising only $18 \%$ of the 
Table 1. Fundamental mass ratio comparison between the endoEarth (lower mantle plus core) and the Abee enstatite chondrite.

\begin{tabular}{|c|c|c|}
\hline Fundamental Earth Ratio & $\begin{array}{l}\text { Earth Ratio } \\
\text { Value }\end{array}$ & $\begin{array}{l}\text { Abee Ratio } \\
\text { Value }\end{array}$ \\
\hline $\begin{array}{l}\text { lower mantle mass to total core mass } \\
\text { inner core mass to total core mass }\end{array}$ & $\begin{array}{l}1.49 \\
0.052\end{array}$ & $\begin{array}{l}1.43 \\
\text { theoretical } \\
0.052 \text { if } \mathrm{Ni}_{3} \mathrm{Si} \\
0.057 \text { if } \mathrm{Ni}_{2} \mathrm{Si}\end{array}$ \\
\hline inner core mass to endo-Earth mass & 0.021 & 0.021 \\
\hline
\end{tabular}

mass of the Earth, later was discovered to have several seismic boundaries, reminiscent of layers of veneer. So the question that faced geoscientists, who believed that the Earth is like an ordinary chondrite meteorite and that the mantle is of uniform chemical composition, is how can those seismicallyindicated layers be explained?

Unless reflected, earthquake waves change speed and direction in a major way for just two reasons: They enter a zone at an angle with either a different crystal structure or a different chemical composition. The assumption by Birch and others, that the mantle is of uniform chemical composition, leads to the only explanation possible, namely, that the seismic boundaries arise from changes in crystal structure caused by the pressure exerted by the weight of the rock above.

Consider this logical exercise: If the inner core is in fact the compound nickel silicide, as suggested by Herndon (1979), then the Earth's core must be like the alloy portion of an enstatite chondrite. If the Earth's core is in fact like the alloy portion of an enstatite chondrite, then the Earth's core should be surrounded by a silicate-rock shell like the silicate-rock portion of an enstatite chondrite. But, the enstatite chondrite type of silicate-rock is essentially devoid of iron combined with oxygen $(\mathrm{FeO})$, unlike the silicate-rock of the upper part of the upper mantle, which has appreciable $\mathrm{FeO}$ (Harte, 1978). This enstatite-chondrite-like silicate-rock shell, if it exists, thus should be bounded by a seismic "discontinuity", the boundary where earthquake waves change speed and direction because of the different compositions. So now here is a prediction which can be tested.

Using the alloy to silicate-rock ratio of the Abee enstatite chondrite (Keil, 1968) and the mass of the Earth's core, by simple ratio proportion Herndon calculated the mass of that enstatite-chondrite-like silicate-rock mantle shell. From tabulated mass distributions (Dziewonski and Gilbert, 1972), he then found the radius of that predicted seismic boundary lies within about $1.2 \%$ of the radius of the major seismic discontinuity which separates the lower mantle from the upper mantle. That logical exercise led to the discovery of fundamental quantitative mass ratio relationships connecting the interior parts of the Earth with parts of the Abee enstatite chondrite (Herndon, 1980, 1998, 2005b), which are shown in Table 1.

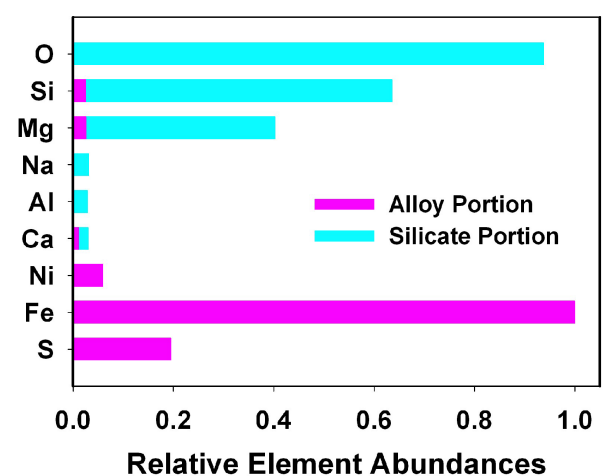

Figure 4. Relative abundances of the major and minor elements in the Abee enstatite chondrite, normalized to iron, showing their relative amounts in the alloy and silicate portions. Note that calcium $(\mathrm{Ca})$, magnesium $(\mathrm{Mg})$, and silicon $(\mathrm{Si})$, normally lithophile elements, occur in part in the alloy portion.

Herndon not only showed that the inner $82 \%$ of the mass of the Earth (the lower mantle and core), called the endo-Earth, is like an enstatite chondrite meteorite, but also showed that the Earth is not like an ordinary chondrite as had been long believed. Metal-bearing chondrites generally consist of three components: nickel-iron alloy, iron sulfide, and silicates. If one were to heat either an ordinary chondrite or an enstatite chondrite to a sufficiently high temperature, the iron metal and iron sulfide would liquefy and combine, settling beneath the less-dense silicate part, just as steel settles beneath slag on a steel-hearth. The Earth is like a spherical steel-hearth, its entire core or alloy part comprising $32.5 \%$ of the planet's mass. Some enstatite chondrites have a sufficiently high percentage of iron-alloy to make such a massive core; but no ordinary chondrites do (Herndon, 1980, 1998, 2005b).

Dahm (1934) and Bullen (1946) first discussed the possibility of some seismic irregularity at the boundary between the core and the lower mantle. Subsequent investigations confirmed the existence of "islands" of matter at the boundary of the core (Lay and Helmberger, 1983; Vidale and Benz, 1993). Just like the seismic features of the upper portion of the mantle, those at the core-mantle boundary have been ascribed to a pressure-induced change in crystal structure (Mao et al., 2004; Murakami et al., 2004). By contrast, Herndon $(1993,1998,2005 b)$ has shown that "islands" of matter at the core-mantle boundary are readily understandable in a logical and causally related way as low-density, high-temperature precipitates from the Earth's enstatite-chondrite-like core.

Figure 4 presents the relative abundances of major and minor elements of the Abee enstatite chondrite, normalized to iron, showing their distribution between silicate and alloy portions. In the more oxygen-rich ordinary chondrites, all of the silicon, calcium and aluminum would occur as oxides in the silicate portion. But, matter like that of the Abee enstatite chondrite formed under conditions that severely 


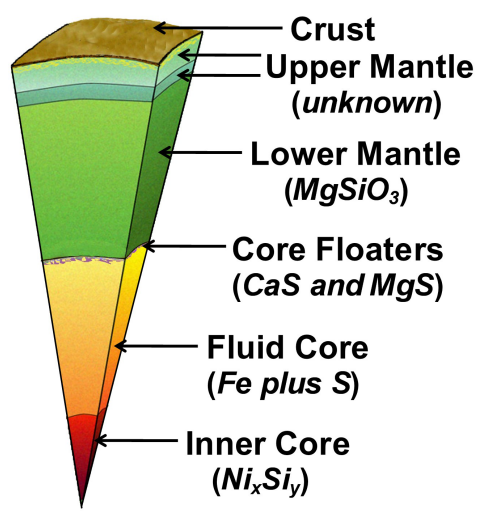

Figure 5. Chemical compositions of the major parts of the endoEarth. From Herndon (2008).

limited available oxygen. Consequently, the alloy portion of enstatite chondrites consists of silicon-containing, nickeliron metal, iron sulfide (FeS), magnesium sulfide $(\mathrm{MgS})$ and calcium sulfide $(\mathrm{CaS})$.

Imagine the Earth's core being comprised of enstatitechondrite-like alloy and being so hot that all of those elements will have dissolved into the iron-based alloy. What happens as this alloy begins to cool? Some elements, such as sulfur, are compatible with being dissolved in molten iron and they will tend to stay dissolved as long as thermodynamically possible. But, some other elements are quite incompatible, particularly oxyphile elements. Incompatible elements, like calcium and magnesium, in a cooling iron solution will seek a way to exsolve, and they find it by combining with sulfur. Both calcium sulfide $(\mathrm{CaS})$ and magnesium sulfide $(\mathrm{MgS})$ form solids at temperatures that are well above the melting point of iron. Both are less dense than iron and will float atop the molten iron. Herndon (1993, 1998, $2005 \mathrm{~b})$ has suggested that calcium sulfide (CaS) and magnesium sulfide (MgS) precipitated from the Earth's core and floated to its top, causing the seismic "roughness" at the coremantle boundary. The observed abundances are appropriate and, moreover, there is an industrial process which is quite similar. Sulfur impurity can weaken steel. So, to remove sulfur from high-quality steel, magnesium or calcium is injected into the molten iron to combine with the sulfur at a high temperature and float to the surface (Foster et al., 1974; Inoue and Suito, 1994; Ribound and Olette, 1978).

Figure 5 is a schematic representation of the chemical composition of the various major parts of the endo-Earth, which is comprised of the lower mantle and core. The compositions of the layers of the upper mantle are yet unknown, but may include components from ordinary and carbonaceous chondrite matter. Note that the chemical compositions of deep-Earth components arise as a consequence of the endo-Earth (the lower mantle plus core) having separated from solar matter under conditions that severely limited available oxygen, which yielded profound differences, with respect to what might have been had the Earth been like an ordinary chondrite. A further example, as discussed in Sections 6 and 8 , is the trace element, uranium, which occurs almost exclusively in the alloy portion of the Abee meteorite and, hence, in the Earth's core.

One note of historical interest: In 1862, Nevil StoryMaskelyne (1823-1911) discovered, in one of the enstatitemeteorites, a mineral of composition $\mathrm{CaS}$, which he named oldhamite in honor of Thomas Oldham (1816-1878), the first Director of the Geological Survey of India (StoryMaskelyne, 1862, 1870). In 1906, Thomas Oldham's son, Richard, discovered the Earth's core (Oldham, 1906). It seems an appropriate tribute that Richard Oldham's core is surrounded by "islands" of oldhamite, named to honor his father.

France Birch, and those who followed him, believing that the Earth's inner core consists of partially crystallized nickeliron metal, plodded optimistically forward, eagerly looking toward the future, but rarely, if ever, looking with question at circumstances in the past which might not hold the same degree of validity as originally thought. As a consequence, their logical progression of understanding and discovery came to a halt. Although nearly four decades had elapsed, J. Marvin Herndon, with an understanding of science history, returned to that same logical juncture and set about following anew the logical progression of understanding and discovery beginning with the idea that Earth's inner core consists, not of partially crystallized nickel-iron metal, but of fully crystallized nickel silicide. The consequence was discovering that the endo-Earth, the inner $82 \%$ of Earth, is like an enstatite chondrite, which not only led to understanding the composition of its components in a logical, causally related manner, but as well, to connecting endo-Earth matter with processes operant during the formation of the Solar System (Sect. 5), which might only have been possible with an appreciation for science history, and, further, to a new understanding of the origin of geomagnetic and planetary magnetic fields (Sects. 8 and 9) and to a new understanding of whole Earth dynamics (Sect. 10).

\section{Progressing further: protoplanetary formation of Earth}

For a time, especially in the early 1950s, planetary formation from hot gaseous protoplanets was discussed (Kuiper, 1951b, a; Urey, 1952), but emphasis changed abruptly with the publication by Cameron (1963) of his diffuse solar nebula models at pressures of about $10^{-5}$ bar, which became so popular as to be called "standard model". According to Beer et al. (2004), "This standard model for the solar system (Mizuno, 1980; Stevenson, 1982; Wetherill, 1980) assumes that planets form initially through the agglomeration of dust into grains, pebbles, rocks and thence planetesimals within a gaseous disc, that these planetesimals coalesce to 
form planetary cores, and that finally (for the giant planets) these cores use gravity to accrete gas from the ever-present disc." For the terrestrial planets, such a model also leads to the further assumption of a "magma ocean", whole-planet melting wherein iron would drain to the planet center forming the core (Birch, 1965; Wood et al., 2006).

The American statistician, George E. P. Box, has stated this about models: "Essentially, all models are wrong, but some are useful" (Box, 1987). So, the question becomes how can one determine the nature of Solar System formation with reasonable certainty without engaging in modelmaking? First, while it is difficult (some might say impossible) to prove that which is true in the natural sciences, one can prove that which is false. Second, rather than making assumptions, one should proceed by securely anchoring ideas and observations to the properties and behavior of matter and radiation. Third, have a deep and broad understanding of relevant science history for therein one might find crucial components necessary for a logical progression of understanding.

During the late 1960s and early 1970s, the so-called "equilibrium condensation" model was developed as part of the above referenced, so-called standard model (Larimer, 1967). That condensation model was based upon the assumption that the mineral assemblage characteristic of ordinary chondrite meteorites, thought to be like the interior of Earth, formed as condensate from a gas of solar composition at pressures of about $10^{-5} \mathrm{bar}$, ultimately becoming planets. The problem with the so-called equilibrium condensation model and the so-called standard model is that both are wrong: First, the interior of Earth is like an enstatite chondrite, not like an ordinary chondrite. Second, from thermodynamic considerations, condensation from solar matter at low pressures, such as $10^{-5}$ bar, would yield a highly oxidized condensate with virtually no metallic iron (Herndon, 1978; Herndon and Suess, 1977), which would lead to the terrestrial planets having insufficiently massive cores, a profound contradiction to what is observed.

On the basis of thermodynamic considerations, Herndon and Suess (1976) showed at the high-temperatures for condensation at high-pressures, solar matter is sufficiently reducing, i.e., it has a sufficiently low oxygen fugacity, for the stability of some enstatite chondrite minerals. However, formation of enstatite-chondrite-like condensate would necessitate thermodynamic equilibria being frozen-in at near-formation temperatures, a puzzling circumstance indeed, unless one understands well the relevant science history: In 1944, the German physicist/chemist, Arnold Eucken (1884-1950), on the basis of thermodynamic considerations, suggested coreformation in the Earth as a consequence of successive condensation from solar matter, on the basis of volatility, from the central region of a hot, gaseous protoplanet with molten iron metal first raining out at the center (Eucken, 1944). Not surprisingly, because the endo-Earth is like an enstatite chondrite, the calculations of Herndon and Suess (1976) complement those of Eucken (1944).
Eucken's concept of Earth raining out on the basis of volatility from the central region of a giant gaseous protoplanet not only solves the long-standing problem of core formation (Birch, 1965; Wood et al., 2006), but, when coupled with another insight from science history, sheds light on the question of why continental rock comprises only about $41 \%$ of the Earth's surface. In 1933, the German engineer/scientist, Ott Christoph Hilgenberg (1896-1976), published his observation that, on a sphere with smaller radius than Earth's present radius, the continents would more-orless fit together like pieces of a jigsaw puzzle, forming a uniform, contiguous shell, before subsequent volumetric Earth expansion led to the formation of the ocean basins (Hilgenberg, 1933).

The protoplanetary mass of Earth, calculated from solar abundances (Anders and Grevesse, 1989) is nearly identical to the mass of Jupiter. Herndon (2006b, 2009c) has shown that the mean density of Earth, compressed to having a surface area $41 \%$ of the present value, is quite similar to the mean density at the gas-rock boundary within the interior of Jupiter (Podolak and Cameron, 1974; Stevenson and Salpeter, 1976). The idea that at an early time the Earth was a gas-giant planet like Jupiter is certainly consistent with observations of near-to-star giant gaseous planets in other planetary systems (Fischer and Valenti, 2005; Santos et al., 2003). Geodynamical implications are discussed in Sect. 10.

\section{Another invisible path at logical juncture: giant planet internal energy production}

In the late 1960s, astronomers discovered that Jupiter radiates into space about twice the energy it receives from the Sun. Later, Saturn and Neptune were also found to radiate prodigious quantities of internally generated energy. That excess energy production has been described by Hubbard (1990) as being "one of the most interesting revelations of modern planetary science." Stevenson (1978), discussing Jupiter, stated, "The implied energy source ... is apparently gravitational in origin, since all other proposed sources (for example, radio-activity, accretion, thermonuclear fusion) fall short by at least two orders of magnitude..." Similarly, more than a decade later, Hubbard (1990) asserted, "Therefore, by elimination, only one process could be responsible for the luminosities of Jupiter, Saturn, and Neptune. Energy is liberated when mass in a gravitationally bound object sinks closer to the center of attraction... potential energy becomes kinetic energy..."

Often to make new discoveries, one must have a broadbased understanding of science history. More than two decades after the discovery of Jupiter's excess internal energy production, with knowledge of the prediction by Kuroda (1956) of the possibility nuclear fission chain reactions in ancient uranium-ore veins, the discovery of the fossil natural nuclear reactors at Oklo (Bodu et al., 1972), the existence 
of uranium in the alloy portion of the Abee meteorite (Murrell and Burnett, 1982), the Earth's interior being like an enstatite chondrite (Herndon, 1980), and more, Herndon (1992) applied the nuclear reactor theory of Fermi (1947) to demonstrate the feasibility of planetocentric natural nuclear fission reactors as energy sources for the giant planets. Revealing that missed logical juncture made it possible to begin a new logical progression of discovery and understanding, which is described further in Sections 8 and 9.

\section{Historical misunderstanding: physical impossibil- ity of Earth-core convection and Earth-mantle convection}

Science history and discovery are inseparable, not only because sometimes logical junctures in the past might have been missed, but because sometimes mistakes are made and built upon. Coming to understand the logical progression of understanding through time in some instances may help one to discover and correct past mistakes.

Moving charges produce magnetic fields (Oersted, 1820). In 1939, the German-American physicist, Walter M. Elsasser (1904-1991), proposed that the Earth's magnetic field is produced by a convection-driven dynamo mechanism in the Earth's fluid core (Elsasser, 1939, 1946, 1950). Since that time, and until quite recently (Herndon, 2009a), Earth-core convection has been taken for granted.

Similarly, the idea of mantle convection was introduced as early as 1921 by Bull (1921) as a brief suggestion to explain continental drift (Wegener, 1912). In 1930, the British geologist, Arthur Holmes (1890-1965), advanced the concept of mantle convection as a means explaining continental movement, producing drawings not unlike those found in modern geology textbooks (Holmes, 1930, 1931). In the early 1960s, the American oceanographer, Harry H. Hess (1906-1969), incorporated the assumption of Earth-mantle convection as a crucial component for his theory of seafloor spreading (Hess, 1962), which was later merged into, and became a crucial component of, plate tectonics theory.

Chandrasekhar (1957) described convection in the following way: "The simplest example of thermally induced convection arises when a horizontal layer of fluid is heated from below and an adverse temperature gradient is maintained. The adjective 'adverse' is used to qualify the prevailing temperature gradient, since, on account of thermal expansion, the fluid at the bottom becomes lighter than the fluid at the top; and this is a top-heavy arrangement which is potentially unstable. Under these circumstances the fluid will try to redistribute itself to redress this weakness in its arrangement. This is how thermal convection originates: It represents the efforts of the fluid to restore to itself some degree of stability." The clarity of Chandrasekhar's explanation led Herndon (2009a) to think-upon and, later, to discover why convection

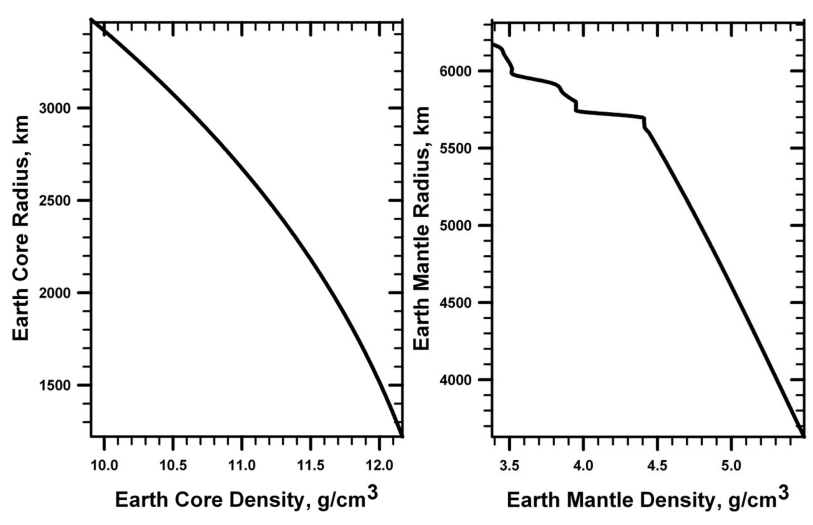

Figure 6. Earth-core and Earth-mantle density as a function of radius. Data from Dziewonski and Anderson (1981) and Dziewonski and Gilbert (1972).

in the Earth's core and in the Earth's mantle is physically impossible.

Calculation of a high Rayleigh Number is often (wrongly) taken as justification for Earth-core convection and for Earthmantle convection. In 1916, Lord Rayleigh (1916) applied the Boussinesq (1903) approximation to Eulerian equations of motion to derive that dimensionless number to quantify the onset of instability in a thin, horizontal layer of fluid heated from beneath. A careful reading of Lord Rayleigh's paper will show that his underlying assumptions, namely, an "incompressible" fluid of "constant" density, except as modified by thermal expansion, and pressure being "unimportant", are inconsistent with the physical parameters of the Earth's core and the Earth's mantle (quotes from Lord Rayleigh (1916)).

Both the Earth's core and the Earth's mantle are compressed by the weight of the mass above (Birch, 1964; Dziewonski and Anderson, 1981; Williamson and Adams, 1923). As shown in Fig. 6, because of its own weight and the weight above, the Earth's core is about is about 23\% denser at the bottom than at the top; likewise, the Earth's mantle is about $62 \%$ denser at the bottom than at the top (Dziewonski and Anderson, 1981; Dziewonski and Gilbert, 1972). The effect of pressure, caused by the weight above, increases density and diminishes the coefficient of thermal expansion (Birch, 1968). The tiny amount of thermal expansion that may occur at the core-bottom, $<1 \%$, cannot overcome the $23 \%$ difference in density between bottom and top, meaning the Earth's core cannot become top-heavy, and meaning also that a thermally expanded "parcel" of bottom-core matter cannot become light enough to float to the core-top, a necessary condition for convection. The Earth's core, therefore, cannot convect. Similarly, the tiny amount of thermal expansion that may occur at the mantle-bottom, $<1 \%$, cannot overcome the $62 \%$ difference in density between bottom and top, meaning the Earth's mantle cannot become topheavy, and meaning also that a thermally expanded "parcel" 


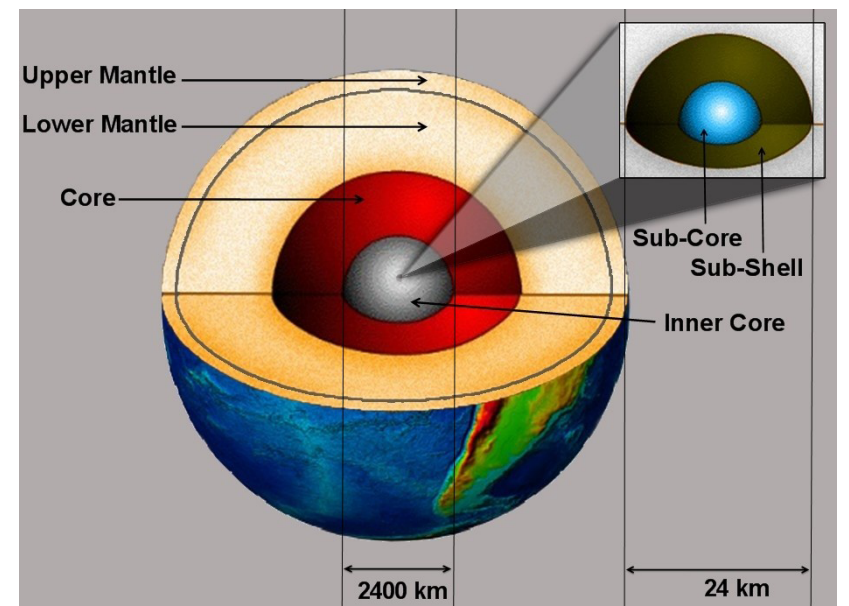

Figure 7. Schematic representation of the major parts of the Earth showing the georeactor at the center in expanded view. From Herndon (2008).

of bottom-mantle matter cannot become light enough to float to the mantle-top, a necessary condition for convection. The Earth's mantle, therefore, cannot convect.

In the natural physical sciences, it is difficult, some might say impossible, to prove a proposition to be true; but, on the other hand, it is quite possible to prove that which is false. Herndon (2009c, a, 2010) has proven the propositions of Earth-core convection and Earth-mantle convection to be false. Thus, all geophysical considerations which are based upon the assumptions of Earth-core convection and Earthmantle convection need to be revised. The implications are highlighted in Sects. 8, 9 and 10.

\section{Progressing still further: origin of the geomagnetic field}

Herndon $(1993,1994)$ applied the nuclear reactor theory of Fermi (1947) to demonstrate the feasibility of a naturally occurring nuclear fission at the center of the Earth, now called the georeactor, as the energy source for the geomagnetic field. In 1996, Herndon (1996) disclosed the sub-structure of the Earth's inner core, describing the two-component structure of the georeactor as consisting of an actinide sub-core, surrounded by a sub-shell composed of the products of nuclear fission and radioactive decay, all surrounded by the inner core, as illustrated in Fig. 7. He also noted the possibility that the sub-shell might be a liquid or slurry.

Hollenbach and Herndon (2001) published the first georeactor numerical simulation conducted using Oak Ridge National Laboratory's SCALE software, which had been validated with nuclear reactor operating data combined with analyses of spent fuel rods (SCALE, 1995). The numerical simulations showed that the georeactor could function over the entire time since Earth's formation, about 4.5 bil-

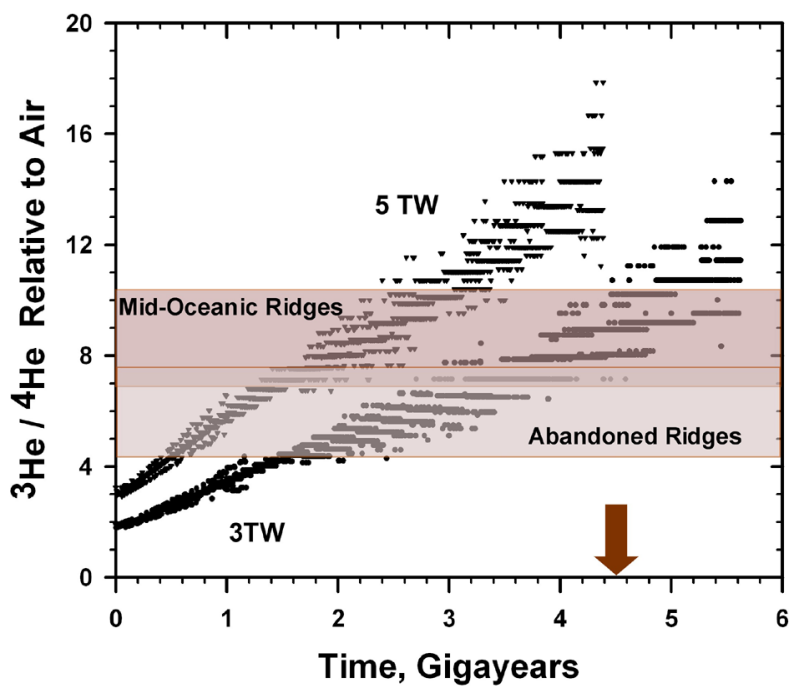

Figure 8. Fission product ratio of ${ }^{3} \mathrm{He} /{ }^{4} \mathrm{He}$, relative to air, from nuclear georeactor numerical simulations at $5 \mathrm{TW}$ (upper) and $3 \mathrm{TW}$ (lower) power levels, from (Herndon, 2003). The bands comprising the $95 \%$ confidence level for measured values from mid-oceanic ridge basalts (MORB) are indicated by the solid lines and shading. The age of the Earth is marked by the arrow. Note the distribution of values at 4.5 gigayears, the approximate age of the Earth. The increasing values are the consequence of uranium fuel burnup. Icelandic "plume" basalts present values ranging as high as 37 (Hilton et al., 1999). Adapted from Herndon (2008).

lion years, as a fast neutron breeder reactor. Moreover, the calculations showed that georeactor-produced ${ }^{3} \mathrm{He}$ and ${ }^{4} \mathrm{He}$ would have the same range of compositions as helium measured in oceanic basalts (Hilton and Porcelli, 2003). Subsequently, Herndon (2003) published more precise numerical simulation data, examples of which are shown in Fig. 8 and provide strong evidence for the existence of Earth's georeactor. The marked, progressive increase in ${ }^{3} \mathrm{He} /{ }^{4} \mathrm{He}$ ratios over time occurs primarily as a consequence of diminished ${ }^{4} \mathrm{He}$ production from radioactive decay as uranium is consumed. Herndon (2003) suggested that the high ${ }^{3} \mathrm{He} /{ }^{4} \mathrm{He}$ ratios observed in Hawaiian and Icelandic lavas (Hilton et al., 1999) portend the demise of the georeactor, although the time scale is uncertain.

Herndon (2007a) presented evidence that the nuclear georeactor fission-product sub-shell is fluid, and suggested that the geomagnetic field is generated within the georeactor subshell, where sustained convection is physically possible, by convection-driven dynamo action, rather that within Earth's iron-alloy fluid core, as had been long thought (Elsasser, 1939, 1950; Glatzmaier, 2002). The implication of the discovery of the physical impossibility of Earth-core convection by Herndon (2009c, a) is quite clear: Either the geomagnetic field is generated by a process other than the convectiondriven dynamo-mechanism, or the geomagnetic field is powered and produced within the georeactor, whose sub-shell can sustain convection for extended periods of time. 
There are profound differences between the idea of geomagnetic field generation by convection-driven dynamo action in the georeactor and the old physically-impossible idea of its being generated within the Earth's fluid core. The radioactive processes of the georeactor provide a plethora of charged particles for producing magnetic seed fields for amplification. Moreover, the mass of the georeactor is less than one ten-millionth that of the fluid core, which means that geomagnetic field changes can take place rapidly, consistent with paleomagnetic observations (Coe and Prevot, 1989).

The geomagnetic field reverses polarity irregularly, north becomes south and vice versa, with an average time between reversals of about 200000 years. In the previous, physicallyimpossible idea of convection-driven dynamo action in the Earth's fluid core, reversals were assumed to be the consequence of some mechanical instability. A different possibility comes to mind as a consequence of extremely low georeactor mass, as compared to the mass of the Earth's fluid core, and by the understanding that the georeactor is part of a magnetic circuit inductively coupled to the charged particle flux of the solar wind.

Stable georeactor operation is assured through establishing a balance between heat-production and actinide settling out. In the micro-gravity environment at the center of Earth, georeactor heat production that is too energetic would be expected to cause actinide sub-core disassembly, mixing actinide elements with neutron-absorbers of the sub-shell, quenching the nuclear fission chain reaction. But as the denser actinide elements begin to settle out of the mix, the chain reaction would re-start, ultimately establishing a balance, a dynamic equilibrium between heat-production and actinide settling-out, a self-regulating control mechanism. Abrupt, ultra-intense changes in the solar wind flux in principle would induce in the georeactor electrical currents, consequently causing ohmic heating, potentially disrupting the georeactor convection pattern and leading to magnetic excursions and to magnetic reversals.

\section{Progressing even further still: planetary magnetic field generation}

Currently active internally generated magnetic fields have been detected in six planets (Mercury, Earth, Jupiter, Saturn, Uranus, and Neptune) and in one satellite (Jupiter's moon Ganymede). Magnetized surface areas of Mars and the Moon indicate the former existence of internally generated magnetic fields in those bodies.

Only three processes, operant during the formation of the Solar System, are responsible for the diversity of matter in the Solar System and are directly responsible for planetary internal-structures, including planetocentric nuclear fission reactors, and for dynamical processes, including and especially, geodynamics. These processes are: (1) Lowpressure, low-temperature condensation from solar matter in the remote reaches of the Solar System or in the interstellar medium, which leads to oxygen-rich condensates; (2) High-pressure, high-temperature condensation from solar matter associated with planetary-formation by raining out from the interiors of giant gaseous protoplanets, which leads to oxygen-starved planetary interiors of enstatite-chondritelike composition, and; (3) Stripping of the primordial volatile components from the inner portion of the Solar System by super-intense solar wind associated with T-Tauri phase massejections, presumably during the thermonuclear ignition of the Sun (Herndon, 2006b).

Herndon $(2009 \mathrm{c})$ generalized the concept of georeactor generation of Earth's magnetic field to other planetary bodies through the commonality of enstatite-chondritelike matter as the primary planet-building material, and through the commonality of georeactor operating conditions at planetary centers, specifically as a self-contained powersource/production-mechanism, in a micro-gravity environment, that functions with the assurance of maintaining an adverse temperature gradient for sustained convection, and for nuclear reactor self-regulation.

\section{Progressing yet even further still: whole-Earth decompression dynamics}

Plate tectonics theory is wrong, being critically dependent upon physically-impossible, assumed mantle convection (Herndon, 2009a). The seemingly compelling agreement between seafloor observations and plate tectonics arises as a consequence of the global dynamics described by Herndon $(2005 a, 2006 a, 2010)$ and called whole-Earth decompression dynamics, which does not require or depend upon mantle convection. Briefly, whole-Earth decompression dynamics is the consequence of Earth having formed as a Jupiter-like gas giant. Beneath about 300 Earth-masses of hydrogen, helium, and other volatile compounds, the silicaterock-plus-alloy kernel was compressed to about $64 \%$ of present-day radius, an amount sufficient to have yielded a closed, contiguous shell of continental rock without ocean basins, called Ottland in honor of Ott Christoph Hilgenberg (1933).

After being stripped of its great overburden of volatile protoplanetary gases by the super-intense $\mathrm{T}$ Tauri-phase solar wind, associated with the thermonuclear ignition of the Sun, the Earth would inevitably begin to decompress, to rebound toward a new hydrostatic equilibrium. The initial wholeEarth decompression is expected to result in a global system of major primary cracks appearing in the rigid crust which persist and are identified as the global, mid-oceanic ridge system, just as explained by Earth expansion theory (Carey, 1976; Hilgenberg, 1933). But here the similarity with that theory ends. Whole-Earth decompression dynamics sets forth a different mechanism for global geodynamics which involves the formation of secondary decompression cracks, 
typically located along continent margins and presently identified as submarine trenches, and the in-filling of those cracks with basalt, extruded from the mid-oceanic ridges, which traverses the ocean floor by gravitational creep, ultimately plunging into and in-filling secondary decompression cracks, thus emulating the plate-tectonic-concept of subduction, but without mantle convection.

As described by Herndon (2010), in whole-Earth decompression dynamics, there is but one true ancient supercontinent, the $100 \%$ closed contiguous shell of continental rock called Ottland in honor of Ott Christoph Hilgenberg (18961976), who first conceived of its existence (Hilgenberg, 1933). Moreover, there can never be another. The successive fragmentation of Ottland may bear only superficial resemblance to the popular, but hypothetical, breakup of Pangaea, where the continents are assumed free to wander, breaking up and re-aggregating, while riding atop non-existent mantle convection cells.

Globally, virtually all major geological activity is the consequence of a single process, the formation of new surface area to accommodate decompression-increased planetary volume, which primarily involves crustal extension fracturing, basalt extrusion, and decompression crack in-filling (Herndon, 2005a, 2006a). The principle driving energy for geodynamics is the stored energy of protoplanetary compression. Many of the explanations of surface phenomena in whole-Earth decompression dynamics are similar to those of plate tectonics, but without mantle convection. Mantle plumes, about which there has been much discussion, involve thermal, not mass, transport, because mantle convection is impossible.

Revealing and correcting mistakes that were made and built upon, like revealing missed logical junctures, generally leads to new logical progressions of understanding and discovery. Correcting the flaws of physically-impossible plate tectonics will inevitably lead to new advances, for example, important new understanding related to petroleum and natural gas exploration (Herndon, 2010).

\section{Yet another invisible path at logical juncture: thermonuclear ignition of stars}

At the beginning of the 20th century, understanding the nature of the energy source that powers the Sun and other stars was one of the most important problems in physical science. Initially, gravitational potential energy release during protostellar contraction was considered, but calculations showed that the energy released would only be sufficient to power a star for a few million years (Helmholtz, 1854; Thomson, 1854), a much shorter time than even the early underestimates of the age of the Earth (Joly, 1899; Thompson, 1890). The discovery of radioactivity and the developments that followed, especially the discovery of nuclear fusion reactions (Oliphant et al., 1934), led to the idea that stars are powered by thermonuclear fusion reactions (Bethe, 1939; Gamow and Teller, 1938).

Thermonuclear fusion reactions are called "thermonuclear" because temperatures on the order of a million degrees Celsius are required. The principal energy released from the detonation of hydrogen bombs comes from thermonuclear fusion reactions. The high temperatures necessary to ignite $\mathrm{H}$-bomb thermonuclear fusion reactions come from their Abomb nuclear fission triggers. Each hydrogen bomb is ignited by its own small nuclear fission A-bomb.

In 1938, when the idea of thermonuclear fusion reactions as the energy source for stars was reasonably well developed (Bethe, 1939), nuclear fission and the nuclear fission chain reaction had not yet been discovered (Hahn and Strassmann, 1939). Astrophysicists assumed that the milliondegree-temperatures necessary for stellar thermonuclear ignition would be produced by the in-fall of dust and gas during star formation and have continued to make that assumption to the present, although clearly there have been signs of potential trouble with the concept. Proto-star heating by the in-fall of dust and gas is off-set by radiation from the surface, which is a function of the fourth power of temperature. Generally, in numerical models of protostellar collapse, thermonuclear ignition temperatures, on the order of a million degrees Celsius, are not attained by the gravitational in-fall of matter without assumption of an additional shockwave induced sudden flare-up (Hayashi and Nakano, 1965; Larson, 1984) or result-optimizing the model-parameters, such as opacity and rate of in-fall (Stahler et al., 1994).

Understanding relevant science history, especially tracing backward in time the logical progression of ideas and discoveries, can lead to new insights and to new discoveries, especially when one recognizes a previously invisible logical juncture. In this instance, the idea of attaining stellar thermonuclear ignition temperatures was initially assumed, in the early 1930s, to result from the in-fall of dust and gas because no other feasible heat source was known. Nuclear fission and the nuclear fission chain reaction had not yet been discovered. After demonstrating the feasibility for planetocentric nuclear fission reactors for the giant, gaseous planets (Herndon, 1992), Herndon (1994) suggested, in a paper published in the "Proceedings of the Royal Society of London", that thermonuclear fusion reactions in stars, as in hydrogen bombs, are ignited by self-sustaining, neutron induced, nuclear fission chain reactions.

As often seems to be the case, when one encounters invisible logical junctures in the past, the resulting new logical progression of understanding and discovery is profoundly different. Previously, since the 1930s, all stars, except brown dwarfs, were thought to ignite automatically upon formation and to be luminous. The idea that stars are ignited by nuclear fission triggers admits the possibility, in the absence of fissionable elements, of stellar non-ignition, a concept which may have fundamental implications bearing on the nature of dark matter (Herndon, 1994), especially the dark matter 
thought to reside in galactic halos and to be responsible for dynamic stability (Rubin, 1983). Moreover, the idea of stellar thermonuclear fusion ignition by nuclear fission provides a new concept for internal heat production in hot Jupiter exoplanets, and for the thermonuclear ignition of galaxies (Herndon, 2008, 2009b).

\section{A new logical progression of understanding and discovery: internal energy production in hot Jupiter exoplanets}

In planetary systems other than our own, some close-tostar, gas-giant exoplanets are called "hot Jupiters" or "puffy Jupiters". Hot Jupiter exoplanets are about as massive as Jupiter, but have anomalously inflated sizes and low densities relative to Jupiter, implying the existence of a powerful internal heat source causing their gaseous envelopes to expand, a circumstance which astrophysicists have been unsuccessful in explaining. For example, in two specific cases, astronomers have suggested, “... there is a source of internal heat that was overlooked by theoreticians" (Charbonneau et al., 2006).

One might expect planetocentric nuclear fission reactors to occur within exoplanets as each has a heavy element component. Indeed, planetocentric nuclear fission reactors may be a crucial component of hot Jupiter exoplanets, but it is unlikely that fission-generated heat alone would be sufficient to create the "puffiness" that is apparently observed. For example, as calculated using Oak Ridge National Laboratory nuclear reactor numerical simulation software, a one Jupitermass solar-composition exoplanet could produce a constant fission-power output of about 400 terawatts (TW) for only about 1/2 billion years (Herndon, 2008, 2009b). Even with that unrealistically short interval of time, the fission-power output of $400 \mathrm{TW}$ is much, much less than the $10000 \mathrm{TW}$ to 10000000 TW required for hot Jupiter "puffiness", according to published hot Jupiter model-estimates (Bodenheimer et al., 2001).

Herndon (2006c, 2009b) has suggested the possibility that hot Jupiter exoplanets may derive much of their internal heat production from thermonuclear fusion reactions ignited by nuclear fission. Unlike stars, hot Jupiter exoplanets are insufficiently massive for gravity to confine thermonuclear fusion reactions throughout a major portion of their gas envelopes. One might anticipate instead thermonuclear fusion reactions occurring at the interface of a central, internal substructure which initially at least was heated to thermonuclear ignition temperatures predominantly by self-sustaining nuclear fission chain reactions. After the onset of fusion at that reactive interface, maintaining requisite thermonuclear-interface temperatures might be augmented by thermonuclear fusionproduced heat, which would as well expand the exoplanetary gas shell, thus decreasing the exoplanet's density. Viewed in this context, hot Jupiter exoplanets appear to be stars in the

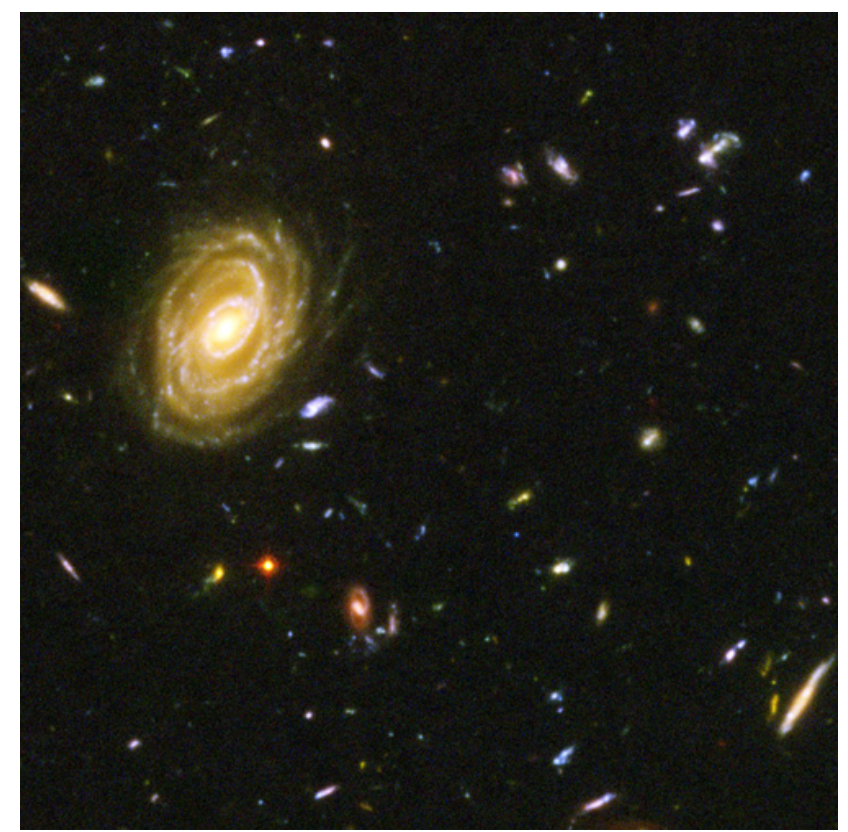

Figure 9. Hubble Space Telescope broad field image showing numerous galaxies.

process of ignition, at the cusp of being a star, but unable to fully ignite because their mass is almost, but not quite, sufficient for gravitational containment. Thus, observations of hot Jupiter exoplanets may stand as the first independent observational evidence for the correctness of Herndon's concept of stellar thermonuclear fusion ignition by nuclear fission chain reactions (Herndon, 1994).

\section{Progressing further: thermonuclear ignition of galaxies}

The concept that thermonuclear fusion reactions in stars are ignited during stellar formation by heat generated by the infall of dust and gas originated before nuclear fission and the nuclear fission chain reaction were discovered and tacitly assumes that all stars more massive than brown dwarfs would automatically ignite. Most stars in the observable Universe appear to be grouped into galaxies that display only a few, frequently-occurring, common morphologies, as shown in Fig. 9, which are wholly inexplicable from the standpoint of stellar ignition by the in-fall of dust and gas. In other words, given that all stars are automatically ignited during formation, how did they become arranged into such a few prominent galactic morphologies?

Observational evidence, primarily based upon velocity dispersions and rotation curves, suggests that spiral galaxies have associated with them massive, spheroidal, dark matter components, thought to reside in their galactic halos $(\mathrm{Ru}-$ bin, 1983) which Herndon (1994) has suggested are composed of Population III stars, zero metallicity stars devoid 


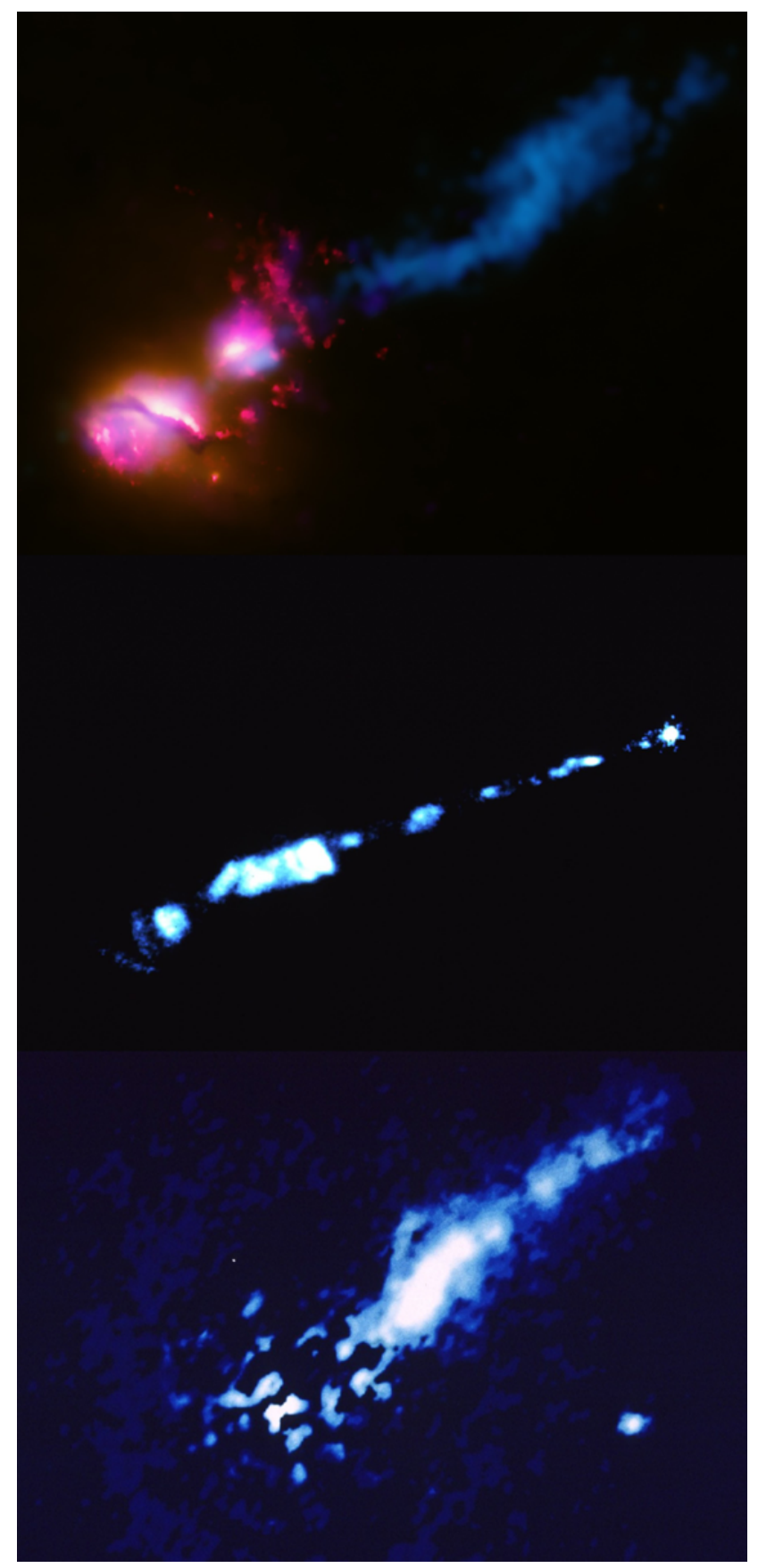

Figure 10. Hubble Space Telescope composite image showing three galactic jets, lengths, top to bottom, 865000 light years, 4000 light years, and 10000 light years.

of fissionable elements, and, consequently, unable to sustain the nuclear fission chain reactions necessary for the ignition of thermonuclear fusion reactions. Interestingly, a one solarmass, Population III dark star would be about the size of Earth (Lynden-Bell and O'Dwyer, 2008).

For half a century, the concept that elements are synthesized within stars (Burbidge et al., 1957) has become widely

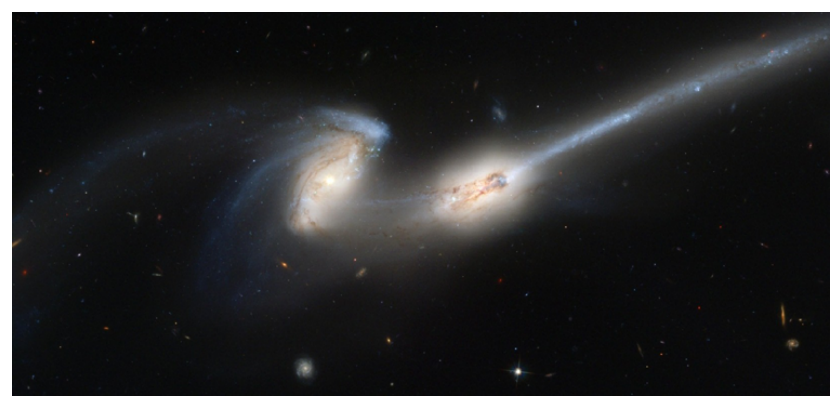

Figure 11. Hubble Space Telescope image of anomalous galaxy NGC 4676.

accepted. In the so-called $\mathrm{B}^{2} \mathrm{FH}$ model, heavy elements are thought to be formed by rapid neutron capture, the R-process, at the supernova end of a star's lifetime; Herndon (2008, 2009b) has suggested another explanation, namely, the formation of highly dense nuclear matter in the galactic core and its associated jetting into space.

The conditions and circumstances at galactic centers appear to harbor the necessary pressures for producing highly dense nuclear matter and the means to jet that nuclear matter out into the galaxy where the jet seeds dark stars which it encounters with heavy elements, including fissionable elements, turning dark stars into luminous stars. Galactic jets, either single or bi-directional, are observed originating from galactic centers, although little is currently known of their nature. Figure 10 is a Hubble Space Telescope composite image of galactic jets, ranging in length from 4000 to 865000 light years.

Consider a more-or-less spherical, gravitationally bound assemblage of dark (Population III) stars, a not-yet-ignited dark galaxy. Now, consider the galactic nucleus as it becomes massive and shoots its first jet of nuclear matter into the galaxy of dark stars, seeding and igniting those stars which it contacts. How might such a galaxy at that point appear? Herndon $(2008,2009 b)$ suggests it would appear quite similar to NGC4676 (Fig. 11) or to NGC10214 (Fig. 12).

The arms of spiral galaxies, such as M101 (Fig. 13), and the bars which often occur in disc galaxies, such as in NGC1300 (Fig. 14), possess morphologies which Herndon (2008, 2009b) suggests occur as a consequence of galactic jetting of nuclear matter containing fissionable elements into the galaxy of dark stars, seeding the dark stars encountered with fissionable elements, thus making possible ignition of thermonuclear fusion reactions.

The structures of just about all luminous galaxies appear to have the jet-like luminous-star features, the imprints of the galactic jets which gave rise to their ignition, the imprints of the distribution of fissionable, heavy element seeds. Therein is the commonality connecting the diverse range of galactic observed structures and the causal relationship which appears to exist. 


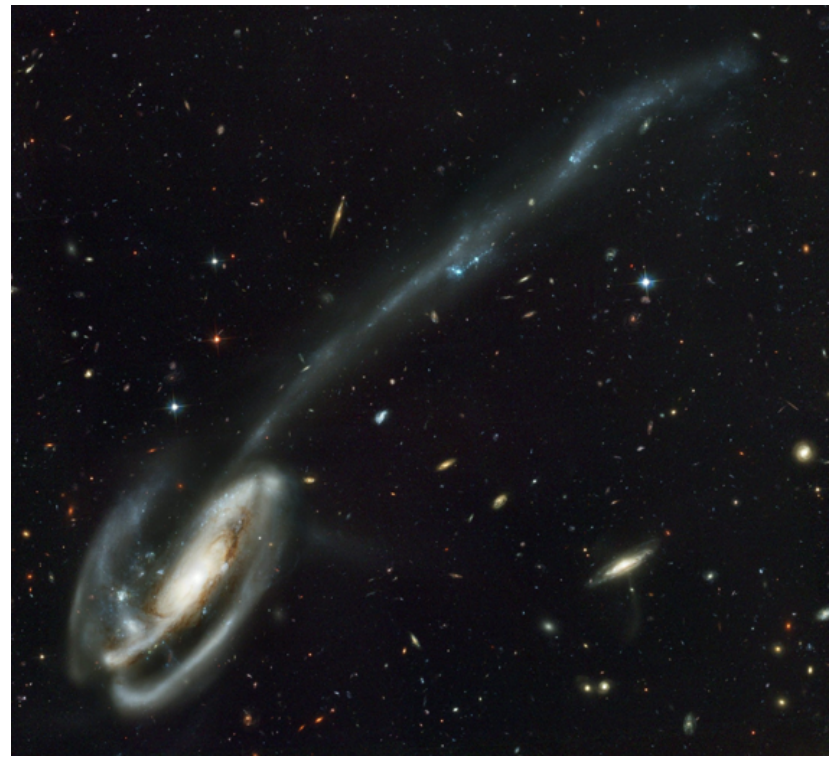

Figure 12. Hubble Space Telescope image of anomalous galaxy, UGC 10214

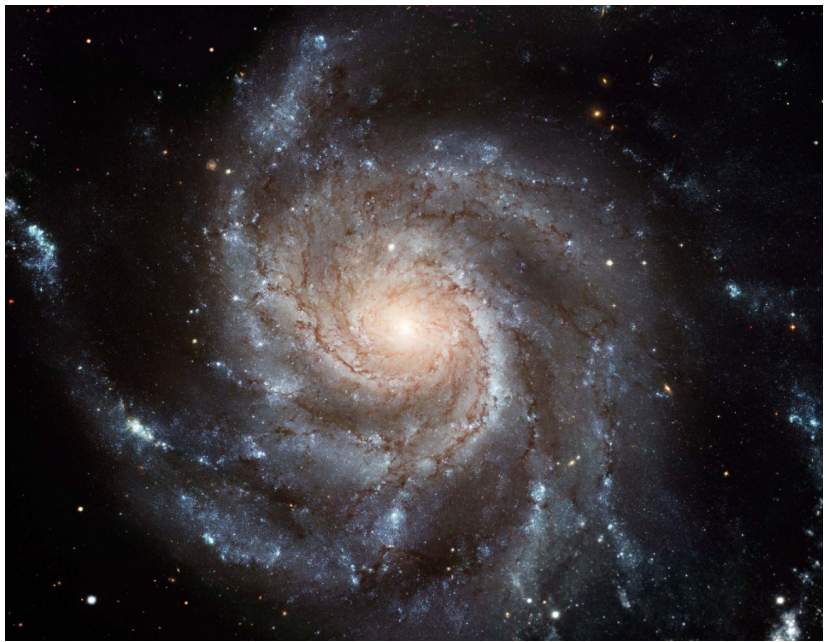

Figure 13. Hubble Space Telescope image of spiral galaxy, M101.

And what of the dark matter necessary for dynamical stability? The dark matter is the spherical halo of un-ignited, dark stars, located just where it must be to impart rotational stability to the galactic luminous structure (Rubin, 1983).

By understanding relevant science history, one can progress backward in time, in a manner of speaking, to discover a formally invisible and missed logical juncture, and, with understanding of the history that had subsequently transpired, can begin anew a different logical progression of ideas, understandings and discoveries. In this instance, one result is realizing that the distribution of luminous stars in a galaxy, and consequently the type of galaxy, for example, barred or spiral, may simply be a reflection of the distribution of the fissionable-elements-containing nuclear matter jetted from the galactic center.

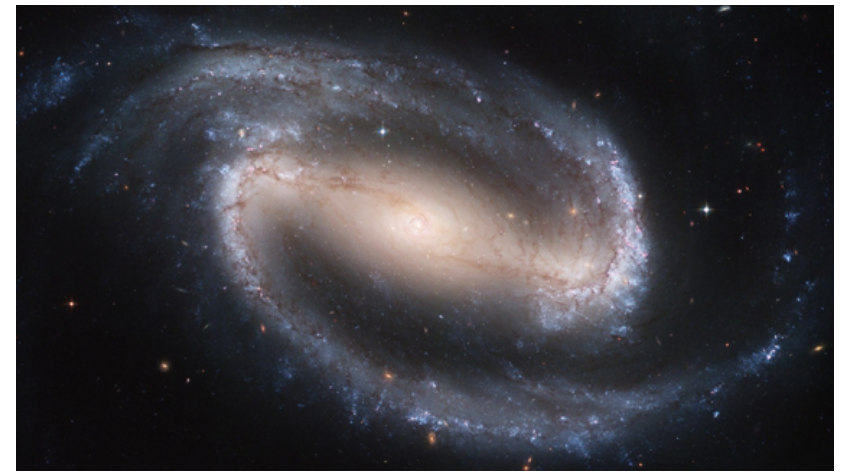

Figure 14. Hubble Space Telescope image of barred spiral galaxy, NGC 1300.

\section{Inseparability of science history and discovery}

The examples presented above should leave little doubt that science history and discovery are indeed inseparable. In the interest of brevity, only the highlights have been presented, and some advances have escaped mention, such as the origin of ordinary chondrite meteorites from a mixture to two primary components (Herndon, 2007b).

Science is an approach toward understanding the true nature of Earth and Universe, a successive progression from the more-unknown to the less-unknown. If Earth and Universe really are as presently perceived, there would be no need for science. The progression of science involves replacing flawed visions of Earth and Universe with less-flawed visions. Science is truly an adventure in understanding and in making scientific discoveries, but to be successful one must be impeccably honest and truthful and must be openly objective to a diverse and broad range of ideas, both in current discussion and in the treasury of science history.

There is a different and more fundamental approach to making scientific discoveries than the frequently discussed variants of the scientific method (Jevons, 1913; Nola and Sankey, 2007; Popper, 2002), and it is this: An individual ponders and through tedious efforts arranges seemingly unrelated observations into a logical sequence in the mind so that causal relationships become evident and new understanding emerges, showing the path for new observations, for new experiments, for new theoretical considerations, and for new discoveries (Herndon, 2008). Science history is rich in "seemingly unrelated observations" just waiting to be logically and causally related to reveal new discoveries.

Edited by: G. P. Gregori

Reviewed by: K. Storetvedt and another anonymous referee 


\section{References}

Aller, L. H.: The Abundances of the Elements, Interscience Publishers, New York, 283 pp., 1961.

Anders, E. and Grevesse, N.: Abundances of the elements: Meteoritic and solar, Geochim. Cosmochim. Acta, 53, 197-214, 1989.

Beer, M. E., King, A. R., Livio, M., and Pringle, J. E.: How special is the Solar System?, arXiv:astro-ph/0407476, 22 July 2004, 2004.

Bethe, H. A.: Energy production in stars, Phys. Rev., 55, 434-456, 1939.

Birch, F.: The alpha-gamma transformation of iron at high pressures, and the problem of the earth's magnetism, Am. J. Sci., 238, 192-211, 1940.

Birch, F.: Elasticity and constitution of the Earth's interior, J. Geophys. Res., 57, 227-286, 1952.

Birch, F.: Density and composition of mantle and core, J. Geophys. Res., 69, 4377-4388, 1964.

Birch, F.: Energetics of core formation, J. Geophys. Res., 70, $6217-$ 6221, 1965.

Birch, F.: Thermal expansion at high pressures, J. Geophys. Res., 73, 817-819, 1968.

Birch, F.: The melting relations of iron, and temperatures in the earth's core, Geophys. J. R. Astron. Soc., 29, 373-387, 1972.

Bodenheimer, P., Lin, D. N. C., and Mardling, R. A.: On the tidal inflation of short-period extrasolar planets, Astrophys. J., 548, 466-472, 2001.

Bodu, R., Bouzigues, H., Morin, N., and Pfiffelmann, J. P.: Sur l'existence anomalies isotopiques rencontrees dan l'uranium gu Gabon, C. R. Acad. Sci., Paris, D275, 1731-1736, 1972.

Boussinesq, J.: Theorie Analytique de la Chaleur, Gauthier-Villars, Paris, 665 pp., 1903.

Box, G. E. P.: Empirical Model-Building and Response Surfaces, Wiley, 1987.

Bull, A. J.: A hypothesis of mountain building, Geol. Mag., 58, 364-367, 1921.

Bullen, K. E.: Note on the density and pressure inside the Earth, Trans. Roy. Soc. New Zealand, 67, 122-124, 1938.

Bullen, K. E.: A hypothesis on compressibility at pressures on the order of a million atmospheres, Nature, 157, p. 405, 1946.

Burbidge, E. M., Burbidge, G. R., Fowler, W. F., and Hoyle, F.: Synthesis of the elements in stars, Rev. Mod. Phys., 29, 547-650, 1957.

Cameron, A. G. W.: Formation of the solar nebula, Icarus, 1, 339342, 1963.

Carey, S. W.: The Expanding Earth, Elsevier, Amsterdam, 488 pp., 1976.

Cavendish, H.: Experiments to determine the density of Earth, Phil. Trans. Roy. Soc. Lond., 88, 469-479, 1798.

Chandrasekhar, S.: Thermal Convection, Proc. Amer. Acad. Arts Sci., 86, 323-339, 1957.

Charbonneau, D., Winn, J. N., Everett, M. E., Latham, D. W., Holman, M. J., Esquerdo, G. A., and O'Donovan, F. T.: Precise radius estimates for the exoplanets WASP-1b and WASP-2b, arXiv.org/astro-ph/0610589, 2006.

Coe, R. S. and Prevot, M.: Evidence suggesting extremely rapid field variation during a geomagnetic reversal, Earth Planet. Sci. Lett., 92, 192-198, 1989.

Copernicus, N.: De revolutionibus orbium coelestium, Johannes Petreius, Nuremberg, 405 pp., 1543.
Dahm, C. G.: A Study of Dilatational Wave Velocity in Earth as a Function of Depth, St. Louis University, St. Louis, MO, 1934.

Daly, R. A.: Meterorites and an earth model, Bull. Geol. Soc. Amer., 54, 401-456, 1943.

Dziewonski, A. M. and Gilbert, F.: Observations of normal modes from 84 recordings of the Alaskan earthquake of 1964 March 28, Geophys. J. R. Astr. Soc., 72, 393-446, 1972.

Dziewonski, A. M. and Anderson, D. A.: Preliminary reference Earth model, Phys. Earth Planet. Inter., 25, 297-356, 1981.

Elsasser, W. M.: On the origin of the Earth's magnetic field, Phys. Rev., 55, 489-498, 1939.

Elsasser, W. M.: Induction effects in terrestrial magnetism, Phys. Rev., 69, 106-116, 1946.

Elsasser, W. M.: The Earth's interior and geomagnetism, Revs. Mod. Phys., 22, 1-35, 1950.

Eucken, A.: Physikalisch-chemische Betrachtungen ueber die frueheste Entwicklungsgeschichte der Erde, Nachr. Akad. Wiss. Goettingen, Math.-K1., 1-25, 1944.

Fermi, E.: Elementary theory of the chain-reacting pile, Science, Wash., 105, 27-32, 1947.

Fischer, D. A. and Valenti, J.: The planet-petallicity correlation, Ap. J., 622, 1102-1117, 2005.

Foster, E., Klapdar, H. W., Richter, H., Rommerswinkel, H. W., Spetzler, E., and Oberhausen, J. W.: Deoxidation and desulphurization by blowing of calcium compounds into molten steel and its effects on the mechanical properties of heavy plates, Stahl u. Eisen, 94, 474-485, 1974.

Fredriksson, K. and Henderson, E. P.: The Horse Creek, Baca County, Colorado, iron meteorite, Trans. Am. Geophys. Un., 46, p. 121, 1965.

Gamow, G. and Teller, E.: The rate of selective thermonuclear reactions, Phys. Rev., 53, 608-609, 1938.

Glatzmaier, G. A.: Geodynamo simulations - How realistic are they?, Ann. Rev. Earth Planet. Sci., 30, 237-257, 2002.

Goldschmidt, V. M.: The principles of distribution of chemical elements in minerals and rocks, Chem. Soc. J., 655-673, 1937.

Goldschmidt, V. M.: Geochemische Verteilungsgesetz der Elemente und der Atom-Artem, Oslo, 1938.

Hahn, O. and Strassmann, F.: Uber den Nachweis und das Verhalten der bei der Bestrahlung des Urans mittels Neutronen entstehenden Erdalkalimetalle, Die Naturwissenschaften, 27, 11-15, 1939.

Harte, B.: Kimberlite nodules, upper mantle petrology, and geotherms, Phil. Trans. R. Soc. Lond., A 288, 487-500, 1978.

Hayashi, C. and Nakano, T.: Thermal and dynamic properties of a protostar and its contraction to the stage of quasi-static equilibrium, Prog. Theor. Physics, 35, 754-775, 1965.

Heath, T. L.: The works of Archimedes, Cambridge University Press, Cambridge, 1897.

Helmholtz, H. v.: On the interaction of natural forces, Phil. Mag., 11, 489-518, 1854.

Herndon, J. M. and Suess, H. E.: Can enstatite meteorites form from a nebula of solar composition?, Geochim. Cosmochim. Acta, 40, 395-399, 1976.

Herndon, J. M. and Suess, H. E.: Can the ordinary chondrites have condensed from a gas phase?, Geochim. Cosmochim. Acta, 41, 233-236, 1977.

Herndon, J. M.: Reevaporation of condensed matter during the formation of the solar system, Proc. R. Soc. Lond, A363, 283-288, 
1978.

Herndon, J. M.: The nickel silicide inner core of the Earth, Proc. R. Soc. Lond, A368, 495-500, 1979.

Herndon, J. M.: The chemical composition of the interior shells of the Earth, Proc. R. Soc. Lond, A372, 149-154, 1980.

Herndon, J. M.: Nuclear fission reactors as energy sources for the giant outer planets, Naturwissenschaften, 79, 7-14, 1992.

Herndon, J. M.: Feasibility of a nuclear fission reactor at the center of the Earth as the energy source for the geomagnetic field, J. Geomag. Geoelectr., 45, 423-437, 1993.

Herndon, J. M.: Planetary and protostellar nuclear fission: Implications for planetary change, stellar ignition and dark matter, Proc. R. Soc. Lond, A455, 453-461, 1994.

Herndon, J. M.: Sub-structure of the inner core of the earth, Proc. Nat. Acad. Sci. USA, 93, 646-648, 1996.

Herndon, J. M.: Composition of the deep interior of the earth: divergent geophysical development with fundamentally different geophysical implications, Phys. Earth Plan. Inter, 105, 1-4, 1998.

Herndon, J. M.: Nuclear georeactor origin of oceanic basalt ${ }^{3} \mathrm{He} /{ }^{4} \mathrm{He}$, evidence, and implications, Proc. Nat. Acad. Sci. USA, 100, 3047-3050, 2003.

Herndon, J. M.: Whole-Earth decompression dynamics, Curr. Sci., 89, 1937-1941, 2005a.

Herndon, J. M.: Scientific basis of knowledge on Earth's composition, Curr. Sci., 88, 1034-1037, 2005b.

Herndon, J. M.: Solar System processes underlying planetary formation, geodynamics, and the georeactor, Earth Moon Planets, 99, 53-99, 2006a.

Herndon, J. M.: Energy for geodynamics: Mantle decompression thermal tsunami, Curr. Sci., 90, 1605-1606, 2006b.

Herndon, J. M.: Nuclear georeactor generation of the earth's geomagnetic field, Curr. Sci., 93, 1485-1487, 2007a.

Herndon, J. M.: Fundamental mass ratio relationships of wholerock chondritic major elements: Implications on ordinary chondrite formation and on planet Mercury's composition, Curr. Sci., 93, 394-399, 2007b.

Herndon, J. M.: Maverick's Earth and Universe, Trafford Publishing, Vancouver, ISBN 978-4251-4132-5, 272 pp., 2008.

Herndon, J. M.: Uniqueness of Herndon's georeactor: Energy source and production mechanism for Earth's magnetic field, arXiv.org/abs/0901.4509, 2009a.

Herndon, J. M.: New concept for internal heat production in hot Jupiter exo-planets, thermonuclear ignition of dark galaxies, and the basis for galactic luminous star distributions, Curr. Sci., 96, 1453-1456, 2009b.

Herndon, J. M.: Nature of planetary matter and magnetic field generation in the solar system, Curr. Sci., 96, 1033-1039, 2009c.

Herndon, J. M.: Impact of recent discoveries on petroleum and natural gas exploration: Emphasis on India, Curr. Sci., 98, 772-779, 2010.

Hess, H. H.: History of Ocean Basins, in: Petrologic Studies: A Volume in Honor of A. F. Buddington, Geological Society of America, Boulder, 599-620, 1962.

Hilgenberg, O. C.: Vom wachsenden Erdball, Giessmann and Bartsch, Berlin, 56 pp., 1933.

Hilton, D. R., Grönvold, K., Macpherson, C. G., and Castillo, P. R.: Extreme ${ }^{3} \mathrm{He} /{ }^{4} \mathrm{He}$ ratios in northwest Iceland: constraining the common component in mantle plumes, Earth Planet. Sci. Lett., 173, 53-60, 1999.
Hilton, D. R. and Porcelli, D.: Noble gases as mantle tracers, in: The Mantle and Core, edited by: Carlson, R. W., Treatise on Geochemistry, Elsevier-Pergamon, Oxford, 277-318, 2003.

Hollenbach, D. F. and Herndon, J. M.: Deep-earth reactor: nuclear fission, helium, and the geomagnetic field, Proc. Nat. Acad. Sci. USA, 98, 11085-11090, 2001.

Holmes, A.: Radioaktivität und Geologie, Verh. Nat. Ges. Basel, 41, 136-185, 1930.

Holmes, A.: Radioactivity and Earth movements, Trans. Geol. Soc. Glasgow 1928-1929, 18, 559-606, 1931.

Hubbard, W. B.: Interiors of the giant planets, in: The New Solar System, edited by: A. Chaikin and J. K. Beatty, Sky Publishing Corp., Cambridge, MA, 134-135, 1990.

Inoue, R. and Suito, H.: Calcium desulfurization equilibrium in liquid iron, Steel Res., 65, 403-409, 1994.

Jeffreys, H.: The Earth, 2 Edn., Cambridge University Press, Cambridge, 345 pp., 1929.

Jevons, W. S.: The Principles of Science: A Treatise on Logic and Scientific Method, Macmillan, London, 786 pp., 1913.

Joly, J.: An estimate of the geological age of the Earth, Trans. Royal Soc., Dublin, VII, 23-66, 1899.

Keil, K.: Mineralogical and chemical relationships among enstatite chondrites, J. Geophys. Res., 73, 6945-6976, 1968.

Kuiper, G. P.: On the origin of the Solar System, Proc. Nat. Acad. Sci. USA, 37, 1-14, 1951a.

Kuiper, G. P.: On the evolution of the protoplanets, Proc. Nat. Acad. Sci. USA, 37, 383-393, 1951b.

Kuroda, P. K.: On the nuclear physical stability of the uranium minerals, J. Chem. Phys., 25, 781-782, 1956.

Larimer, J. W.: Chemical fractionation in meteorites I, Condensation of the elements, Geochim. Cosmochim. Acta, 31, 12151238, 1967.

Larson, R. B.: Gravitational torques and star formation, Mon. Not. R. Astr. Soc., 206, 197-207, 1984.

Lay, T. and Helmberger, D. V.: The shear wave velocity gradient at the base of the mantle, J. Geophys. Res., 88, 8160-8170, 1983.

Lehmann, I.: P', Publ. Int. Geod. Geophys. Union, Assoc. Seismol., Ser. A, Trav. Sci., 14, 87-115, 1936.

Lord Rayleigh: On convection currents in a horizontal layer of fluid where the higher temperature is on the under side, Phil. Mag., 32, 529-546, 1916.

Lynden-Bell, D. and O'Dwyer, J. P.: One mass-radius relation for planets, white dwarfs, and neutron stars, arXiv.org/astroph/0104450, 2008.

Mao, W. L., Shen, G., Prakapenka, V. B., Meng, Y., Campbell, A. J., Heinz, D. L., Shu, J., Hemley, R. J., and Mao, H.: Ferromagnesian postperovskite silicates in the D" layer of the Earth, Proc. Nat. Acad. Sci. USA, 101, 15867-15869, 2004.

Mason, B.: The classification of chondritic meteorites, Amer. Museum Novitates, 2085, 1-20, 1962.

Mizuno, H.: Formation of the Giant Planets, Prog. Theo. Phys., 64, 544-557, 1980.

Murakami, M., Kei, H., Katsuyuki, K., Nagayoshi, S., and Yasuo, O.: Post-perovskite phase transition in $\mathrm{MgSiO}_{3}$, Sci., 304, 855858, 2004.

Murrell, M. T. and Burnett, D. S.: Actinide microdistributions in the enstatite meteorites, Geochim. Cosmochim. Acta, 46, 24532460, 1982. 
Nola, R. and Sankey, H.: Theories of Scientific Method: An Introduction, Acumen, Chesham, 381 pp., 2007.

Oersted, H. C.: Galvanic magnetism, Phil. Mag., 55, p. 394, 1820.

Oldham, R. D.: The constitution of the interior of the earth as revealed by earthquakes, Q. T. Geol. Soc. Lond., 62, 456-476, 1906.

Oliphant, M. L., Harteck, P., and Rutherford, E.: Transmutation effects observed with heavy hydrogen, Nature, 133, p. 413, 1934.

Podolak, M. and Cameron, A. G. W.: Models of the Giant Planets, Icarus, 22, 123-148, 1974.

Popper, K. R.: Conjectures and Refutations: The Growth of Scientific Knowledge, Routledge, London, 593 pp., 2002.

Ramdohr, P. and Kullerud, G.: Stony Meteorites, Carnegie Institution of Washington Year Book, 61, 163-165, 1962.

Ramdohr, P.: Einiges ueber Opakerze im Achondriten und Enstatitachondriten, Abh. D. Akad. Wiss. Ber., Kl. Chem., Geol., Biol., 5, 1-20, 1964.

Ramdohr, P.: The Opaque Minerals in Stony Meteorites, Elsevier, New York, 245 pp., 1973.

Reed, S. J. B.: Perryite in the Kota-Kota and South Oman enstatite chondrites, Mineral. Mag., 36, 850-854, 1968.

Ribound, P. and Olette, M.: Desulfurization by alkaline-earth elements and compounds, Physical Chemistry and Steelmaking, Versailles, France, 1978.

Ringwood, A. E.: Silicon in the metal of enstatite chondrites and some geochemical implications, Geochim. Cosmochim. Acta, $25,1-13,1961$.

Rubin, V. C.: The rotation of spiral galaxies, Sci., 220, 1339-1344, 1983.

Santos, N. C., Israelian, G., Mayor, M., Rebolo, R., and Udry, S.: The HARPS survey for southern extra-solar planets II. A 14 Earth-masses exoplanet around mu Arae, Astron. Astrophys., 426, L19-L23, 2003.

SCALE: A Modular Code System for Performing Standardized Analyses for Licensing Evaluations, N. C., Rev. 4, (ORNL/NUREG/CSD-2/R4), Vols. I, II, and III, April 1995, available from Radiation Safety Information Computational Center at Oak Ridge National Laboratory as CCC-545, 1995.

Scott, E. R. D.: Chondrites and the protoplanetary disk, Ann. Rev. Earth Planet. Sci., 35, 577-620, 2007.

Stahler, S. W., Korycansky, D. G., Brothers, M. J., and Touma, J.: The early evolution of protostellar disks, Astrophys. J., 431, 341$358,1994$.
Stevenson, D. J. and Salpeter, E. E.: Interior models of Jupiter, in: Jupiter: Studies of the Interior, Atmosphere, Magnetosphere, and Satellites, edited by: Gehrels, T., University of Arizona Press, Tucson, 85-112, 1976.

Stevenson, D. J.: Formation of the giant planets, Planet. Space Sci., 30, 755-764, 1982.

Stevenson, D. J.: The outer planets and their satellites, in: The Origin of the Solar System, edited by: Dermott, S. F., Wiley, New York, 395-431, 1978.

Stimson, D.: The Gradual Acceptance of the Copernican Theory of the Universe, Faculty of Political Science, Columbia University, Hanover, New Hampshire, 149 pp., 1917.

Story-Maskelyne, N. S.: On aerolites, Dept. Brit. Ass. Advanc. Sci., 32, 188-191, 1862.

Story-Maskelyne, N. S.: On the mineral constituents of meteorites, Phil. Trans. Roy. Soc. Lond., 160, 198-214, 1870.

Suess, H. E., and Urey, H. C.: Abundances of the Elements, Rev. Mod. Phys., 28, 53-74, 1956.

Thompson, W.: On the secular cooling of the Earth, in: Mathematical and Physical Papers' Volume III, Elasticity, Heat, ElectroMagnetism, C. J. Clay and Sons, London, 295-311, 1890.

Thomson, W.: On the mechanical energies of the Solar System, Phil. Mag., 8, 409-430, 1854.

Urey, H. C.: The Planets, Yale University Press, New Haven, 1952.

Vidale, J. E. and Benz, H. M.: Seismological mapping of the fine structure near the base of the Earth's mantle, Nature, 361, 529532, 1993.

Wai, C. M.: The metal phase of Horse Creek, Mount Egerton and Norton County enstatite meteorites, Mineral. Mag., 37, 905-908, 1970.

Wasson, J. T. and Wai, C. M.: Composition of the metal, schreibersite and perryite of enstatite achondrites and the origin of enstatite chondrites and achondrites Geochim. Cosmochim. Acta, 34, 169-184, 1970.

Wegener, A. L.: Die Entstehung der Kontinente, Geol. Rundschau, 3, 276-292, 1912.

Wetherill, G. W.: Formation of the terrestrial planets, Ann. Rev. Astron. Astrophys., 18, 77-113, 1980.

Wiechert, E.: Ueber die Massenverteilung im Inneren der Erde, Nachr. K. Ges. Wiss. Goettingen, Math.-K1., 221-243, 1897.

Williamson, E. D. and Adams, L. H.: Density distribution in the Earth, J. Wash. Acad. Sci., 13, 413-428, 1923.

Wood, B. J., Walter, M. J., and Wade, J.: Accretion of the earth and segregation of its core, Nature, 441, 825-833, 2006. 